\title{
KIDNEY CARCINOMA ASSOCIATED WITH XP11.2 TRANSLOCATION / TFE3 (ASPL-TFE3) GENE FUSION
}

\author{
PAULO G. O. SALLES, MARIO SOTO JR
}

Biocor Hospital, Belo Horizonte, Minas Gerais, Brazil

\begin{abstract}
We report the case of a 58-year old patient, showing a solid image in the right kidney, who underwent radical nephrectomy that revealed neoplasia, whose pathological study led to the diagnosis of kidney carcinoma associated with Xp11.2 translocation / TFE3 (ASPL-TFE3) gene fusion. The authors discuss aspects related to this lesion, such as frequency, pathogenesis, clinical presentation, histopathology and outcome, as observed in the literature.
\end{abstract}

Key words: kidney neoplasms; renal cell; nephrectomy; TFE3 protein, human

Int Braz J Urol. 2005; 31: 251-5

\section{INTRODUCTION}

Kidney carcinomas associated with Xp11.2 translocations / TFE3 gene fusions are present in the new version of the WHO classification of kidney tumors (2004) (1). They are defined by a number of different translocations involving the Xp11.2 chromosome, all of them resulting from genic fusions involving the TFE3 gene. It is an uncommon tumor, with approximately 30 reports in international literature, with no Brazilian publications, whose morphology and biological behavior are not widely recognized as yet (1-3).

\section{CASE REPORT}

Female 58-year old patent reported infrequent episodes of nephritic colic during the past 6 months. The physical examination revealed no significant changes, as well as the exam of urinary sediment and urine culture. Imaging examinations (Figure-1) showed a nodule in the middle third of right kidney. Considering this finding, after a discussion on surgical approach and management with the patient, we opted for a right radical nephrectomy. The surgical procedure was performed without any intercurrence, and immediate post-operative outcome was satisfactory.

The pathological examination showed right kidney measuring $12.5 \times 7.5 \times 5.5 \mathrm{~cm}$, weighting $242 \mathrm{~g}$, and presenting in its middle portion, in subcapsular position, a circumscribed nodular lesion measuring $4.8 \times 4.5 \times 3.5 \mathrm{~cm}$. When observing the sections, the lesion was well delimited, with brownish-yellowish color, containing areas of necrosis and hemorrhage. It presented a varying aspect, in a combination of cystic, solid and papilliferous areas (Figure-1). The renal pelvis was not affected, and renal artery and vein, as well as the ureter, showed no signs of neoplastic infiltration. The tumor did not pass over the renal capsule. Six lymph nodes from the perirenal fibroadipous tissue were dissected, with sizes ranging from 0.6 to $2 \mathrm{~cm}$ in the longest axis. On microscopy, the tumor showed either papillary or solid architecture (forming well defined cell nests), hyaline nodules and cells with clear or slightly eosi- 


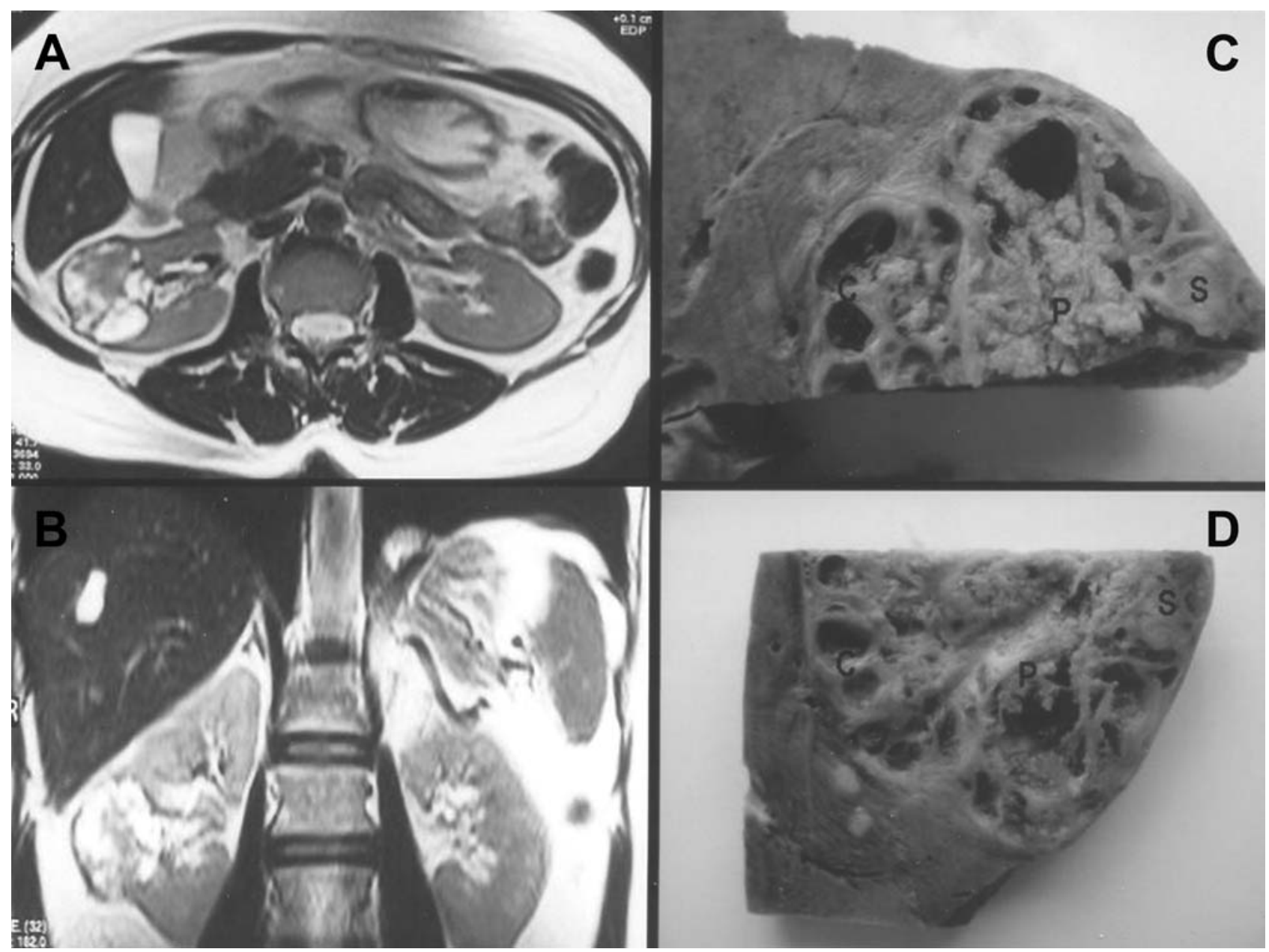

Figure 1 - A) Magnetic resonance imaging depicting the lesion in right kidney, in transversal and B) frontal sections. C) and D) Details from the macroscopic examination, showing varied aspect, with cystic $(C)$, papillary $(P)$ and solid $(S)$ areas.

nophilic and granular cytoplasm, limited by quite distinctive edges (Figure-2). Metastases were seen in 3 of the 6 dissected lymph nodes, thus determining a final staging (TNM 2002) pT1b pN2 pMX (stage IV). Based on the aforementioned morphological findings, immunohistochemical analysis was performed in order to search for the TFE3 protein, with positive result. The set of morphological and immunohistochemical findings confirmed the diagnosis of renal cell carcinoma associated with Xp11.2 translocation / TFE3 (ASPL-TFE3) gene fusion. After approximately 6 months of follow-up, the patient shows favorable outcome, without manifesting disease or any other signs or symptoms.

\section{COMMENTS}

These tumors are characterized by translocations involving the Xp11.2 chromosome, all of them resulting in genic fusions involving the TFE3 gene. Those include $\mathrm{t}(\mathrm{X} ; 1)(\mathrm{p} 11.2 ; \mathrm{q} 21)$ translocation, with fusion of the TFE3 and PRCC genes, a $\mathrm{t}(\mathrm{X} ; 1)(\mathrm{p} 11.2 ; \mathrm{p} 34)$ translocation, with fusion between PSF and TFE3, inv $(X)(p 11 ; q 12)$ translocation, with fusion of NonO (p54 $4^{\text {nrb }}$ ) and TFE3 genes, and $\mathrm{t}(\mathrm{X} ; 17)(\mathrm{p} 11.2 ; \mathrm{q} 25)$ translocation, with fusion of the ASPL (also known as RCC17 or ASPSCR1) and TFE3 genes. The latter is also present, though in a cytogenetically unbalanced form, in alveolar soft part sar- 


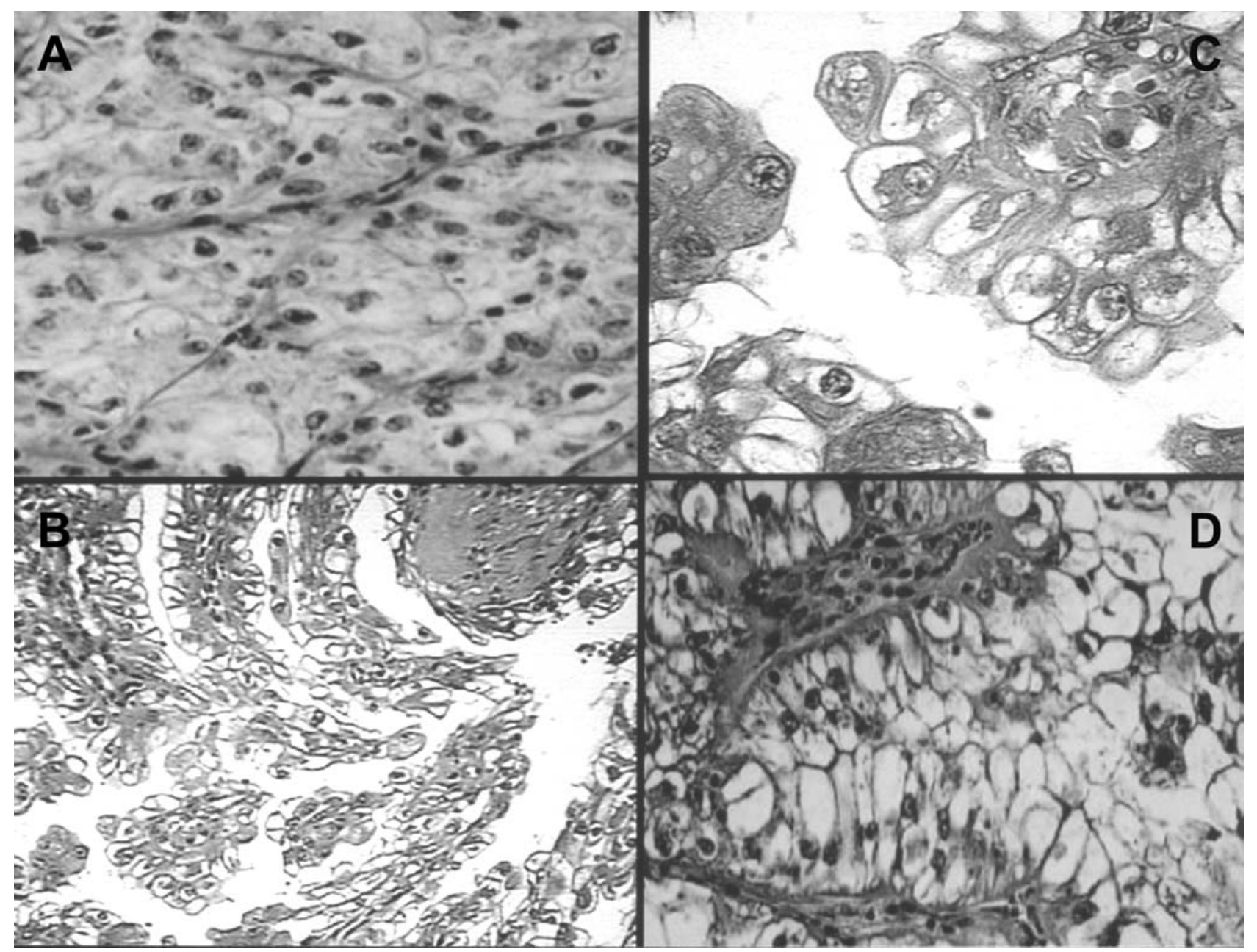

Figure 2 - Sequence of photomicrographies. A) Solid tumoral area, containing cells with clear, finely granular cytoplasm (HE, X200). $B), C)$ and $D)$ Show papillary areas at increasing magnifications, highlighting the hyaline nodule (B) and the cytoplasm aspect, which is clear/finely granular with well-defined membrane (C) (HE, X100, X200 and X400, respectively).

comas (1-3). A chromosome translocation is an aberration associated with a change in the chromosomal structure: a segment from one chromosome is transferred to another one. This kind of change allows the recombination of unrelated sequences from different chromosomes, with the formation of hybrid genes. Such genes (then considered as oncogenes), in turn, can eventually encode chimeric (mutant) proteins that would act as mediators related to cell growth and motility, potential for invasion and cellular morphogenetic differentiation. Thus, recognizing and studying such chromosomal change could have a funda- mental role for understanding carcinogenesis, as well as for diagnosing and determining prognostic factors associated with kidney carcinomas $(2,3)$.

These tumors usually occur in children and young adults (on the second and third decades of life), though some cases have been described in older patients as well; apparently there is no difference in the distribution by gender. Morphologically, they are generally characterized by their varied aspect, with predominantly papillary areas, eventually with solid or cystic portions. Cells have a somewhat voluminous, clear or slightly eosinophilic cytoplasm, with well-de- 
fined limits, and nuclei containing vesicular chromatin and prominent nucleoli. Scattered hyaline nodules and psammomatous bodies can be seen. Such findings can vary according to the different variants (ASPL-TFE3 tumors have cells with less abundant cytoplasm, more hyaline nodules and psammomatous bodies, and are less solid than PRCC-TFE3 tumors). The immunohistochemical analysis reveals the nuclear labeling for the chimeric protein TFE3, which is produced by the fusion of genes that encode chimeric proteins composed by the $\mathrm{C}$-terminal portion of TFE3 and by N-terminal portions of other translocated segments, which constitutes the most distinctive feature in these neoplasias; expression of CD10 and RCC (renal cell carcinoma marker antigen) has been described, but only $50 \%$ express epithelial markers (EMA and cytokeratin, for example) (1-4). Differential diagnosis includes papillary and conventional (clear cell) renal cell carcinomas. In relation to outcome, little is known about the clinical behavior of these carcinomas: the literature reports that tumors ASPL-TFE3, despite their advanced stage, usually presented slow progression (1-2). In summary, it is a rare renal tumor, with peculiar morphogenetic characteristics and biological behavior, which must be recognized by specialists. $\overline{\text { Dr. Pedram Argani, Johns Hopkins }}$ University Hospital,Baltimore, MD, USA, performed the immunohistochemical analysis.

\section{REFERENCES}

1. Tumours of the Kidney. In: Eble JN, Sauter G, Epstein JI, Sesterhenn I (eds.). WHO Classification of Tumours: Tumours of the Urinary System and Male Genital Organs. Lyon, IARC Press. 2004, pp. 9-88.

2. Argani P, Antonescu CR, Illei PB, Lui MY, Timmons CF, Newbury R, et al.: Primary renal neoplasms with the ASPL-TFE3 gene fusion of alveolar soft part sarcoma: a distinctive tumor entity previously included among renal cell carcinomas of children and adolescents. Am J Pathol. 2001; 159: 179-92.

3. Argani P, Antonescu CR, Couturier J, Fournet JC, Sciot R, Debiec-Rychter M, et al.: PRCC-TFE3 renal carcinomas: morphologic, immunohistochemical, ultrastructural, and molecular analysis of an entity associated with the $\mathrm{t}(\mathrm{X} ; 1)(\mathrm{p} 11.2 ; \mathrm{q} 21)$. Am J Surg Pathol. 2002; 26: 1553-66.

4. Argani P, Lal P, Hutchinson B, Lui MY, Reuter VE, Ladanyi M. Aberrant nuclear immunoreactivity for TFE3 in neoplasms with TFE3 gene fusions: a sensitive and specific immunohistochemical assay. Am J Surg Pathol. 2003; 27: 750-61.

\author{
Correspondence address: \\ Dr. Paulo G. de Oliveira Salles \\ Rua Pitangueiras 554 \\ Belo Horizonte, MG, 30350-200, Brazil \\ Phone.: + 5531 3296-9851 \\ E-mail: paulogsalles@yahoo.com
}




\section{EDITORIAL COMMENT}

The WHO has recently presented a new classification for renal cell carcinomas in 2004. This classification included renal carcinomas with fusion of the ASPL gene, which is present in alveolar soft part sarcoma alveolar, and the PRCC gene, found in papillary renal cell carcinoma. Jointly, theses tumors have been denominated as carcinomas with translocation of TFE3 gene in Xp 11.2 (PRCC-TFE3 and ASPLTFE3).

Recent information from European pathologists and from the John Hopkins University show that a large percentage of renal carcinomas occurring in the first decades of life present TFE3 translocations, thus suggesting that this group of tumors that are currently described, under a pathological perspective, as typical renal cell carcinomas, are in fact genetically and phenotypically different from conventional tumors.

The case described in this paper is interesting; furthermore, it reports an adult patient, which is an uncommon fact. The real clinical significance of this gene fusion is still unknown, but it could possibly indicate tumors that are sensitive to different chemotherapy agents.

Dr. Marcus V. Sadi

Section of Urology

University of Santo Amaro

Sao Paulo, SP, Brazil 